\title{
Modeling of Excavation Damaged Zone through the strain localization approach in Boom clay
}

\author{
F. Salehnia \& R. Charlier \\ Département ArGEnCo, Institut de Mécanique et Génie Civil, Université de Liège, Liège, Belgium \\ X. Sillen \\ Belgian Nuclear Research Center (SCK-CEN), Mol, Belgium \\ A. Dizier \\ European Underground Research Infrastructure for Disposal of Nuclear Waste in Clay Environment, \\ EIG EURIDICE, Mol, Belgium
}

ABSTRACT: Deep disposal of the high-level nuclear wastes in the potential geological formations is a promising option for the long-lived radioactive waste management. A damaged zone with significant irreversible deformations and important host rock's properties changes is expected to be created around the underground galleries due to the excavation process that this zone is called as Excavation Damaged Zone (EDZ). The excavation process of the Connecting gallery in the Boom clay formation (Mol, Belgium) is analyzed in this study. Since during its construction, the localized crack propagation was observed around the gallery, we propose to analyze the EDZ and its extension around the Connecting gallery in the framework of strain localization approach. Numerical modeling of strain localization needs a specific approach to overcome the practical problem of mesh size dependency within the framework of classical finite elements. Therefore, the second gradient method as an enhanced technique is applied to adjust this problem. This study is aimed to model the coupled hydro-mechanical behavior of the Boom clay host-rock during the construction of the Connecting gallery. A quarter of the gallery is studied by symmetry in the plane strain conditions and under the initial anisotropic stress state providing the information on the evolution of strain localization in shear bands mode accomplished by the extension of EDZ. In addition, considering a concrete lining on the gallery wall, a discussion on the contact pressure on the interface between the massive and the lining is performed simultaneously by the evolution of shearing bands in the vicinity of the gallery.

\section{INTRODUCTION}

Considering the fact that nuclear power is strategically and quantitatively an important contributor to some industries like the global electricity generation, treating some amount of high-level nuclear waste as the inevitable outcome is a significant issue nowadays. Disposing the high-level and long-lived radioactive waste in the potential deep geological stable formation is a promising option in the framework of their longterm management. The depth of a repository (several hundreds of meters) should ensure isolation from the biosphere, while host rock characteristics such as a low permeability is essential so that if ever there is a loss of containment due to the degradation of the engineered barrier system, radionuclides release's rates will remain limited. Clay formations in their natural state mostly exhibit favorable conditions and properties, among which a very low hydraulic conductivity is noticeable. Boom Clay formation located in the northeast of Belgium is studied as one of these potential host-rock formations for the disposal of high-level and long-lived radioactive waste considering its low permeability, large retention (sorption) capacity for many radionuclides and its self-sealing capacity.

The Excavation Damaged Zone (EDZ) as an issue of a particular interest in the context of the long-term management of high-level nuclear waste disposal, is expected as a consequence of the underground gallery excavation in these clay formations. Stress redistribution generated around the gallery may lead to the creation of a zone with significant irreversible deformations and changes of hydro-mechanical properties. Thus, the extension of the damaged zone and the fracturing network in this zone in the underground structure scale remains an essential issue.

The localized rupture was mainly observed as the essential source of failure during the construction of the underground galleries in the Boom clay host-rock. Furthermore, the localization of deformation within the shear bands due to the localized micro-crack propagation is also confirmed in many experiments as the 
classical mode of failure of geomaterials (Lenoir et al. 2007). Therefore, the structure and extension of the EDZ during the construction of a gallery excavation in the deep Boom clay formation in Mol, Belgium is modeled numerically in this study through the strain localization approach.

\section{NUMERICAL APPROACH FOR MODELING OF STRAIN LOCALIZATION}

The numerical modeling of strain localization within the framework of the classical finite element is depending on the mesh size and orientation. Therefore, we need an enhanced method as well as the the classical constitutive mechanical law to model properly the post-peak behavior (Rice 1976, Collin et al. 2009). As the constitutive mechanical law, the Drucker-Prager yield surface given by equation (1) is used in the framework of a frictional elastoplastic model:

$F=I I_{\hat{\sigma}}+m\left(I_{\sigma}-\frac{3 c}{\tan \phi_{c}}\right)=0$,

where $I I_{\hat{\sigma}}$ is the second deviatoric stress invariant defined by equation (3) in which $\hat{\sigma_{i j}}$ is the deviatoric stress tensor and $\sigma_{i j}$ is the total stress field defined according to the Bishop \& Terzaghi's postulate (equation (4)), $I_{\sigma}=\sigma_{i j} \delta_{i j}$ is the first stress invariant, $c$ is the cohesion, $\phi_{c}$ is the compression friction angle, and $\mathrm{m}$ is defined as the following:

$m=\frac{2 \sin \phi_{c}}{\sqrt{3}\left(3-\sin \phi_{c}\right)}$,

$I I_{\hat{\sigma}}=\sqrt{\frac{1}{2} \hat{\sigma_{i j}} \hat{\sigma_{i j}}} ; \hat{\sigma_{i j}}=\sigma_{i j}-\frac{I_{\sigma}}{3} \delta_{i j}$,

$\sigma_{i j}=\sigma_{i j}^{\prime}-S_{r, w} p_{w} \delta_{i j}$,

where $\sigma_{i j}^{\prime}$ is the effective stress, $S_{r, w}$ is the water saturation degree, and $\delta_{i j}$ is the Kronecker symbol.

The friction angle and/or cohesion hardening and/or softening can be occurred as a function of Von Mises equivalent plastic strain brought in equation (5) in which the hyperbolic functions are defined as equations (6)-(9).

$\varepsilon_{e q}^{p}=\sqrt{\frac{2}{3} \varepsilon_{i j}^{\hat{p}} \varepsilon_{i j}^{\hat{p}}}$,

if $\varepsilon_{e q}^{p}<d e c_{\phi}: \phi_{c}=\phi_{c 0}$,

if $\varepsilon_{e q}^{p}>d e c_{\phi}: \phi_{c}=\phi_{c 0}+\frac{\phi_{c f}-\phi_{c 0}\left(\varepsilon_{e q}^{p}-d e c_{\phi}\right)}{B_{\phi}+\left(\varepsilon_{e q}^{p}-\operatorname{dec}_{\phi}\right)}$,

if $\varepsilon_{e q}^{p}<d e c_{c}: c=c_{0}$,

if $\varepsilon_{e q}^{p}>d e c_{c}: c=c_{0}+\frac{c_{f}-c_{0}\left(\varepsilon_{e q}^{p}-d e c_{c}\right)}{B_{c}+\left(\varepsilon_{e q}^{p}-d e c_{c}\right)}$, where $\phi_{c 0}$ is the initial compression friction angle, $\phi_{c f}$ is the final compression friction angle, $c_{0}$ is the initial cohesion, $c_{f}$ is the final cohesion, $\operatorname{dec}_{\phi} / \operatorname{dec}_{c}$ are the values of equivalent plastic strain from which hardening/softening starts, and $B_{\phi} / B_{c}$ are the values of equivalent plastic strain for which half of hardening/softening on friction angle and cohesion is achieved (Barnichon 1998).

Moreover, as it has been already mentioned, a specific approach is needed for correctly modeling the localization phenomenon. Among the different technics, the second gradient model (Chambon et al. 1998, Chambon et al. 2001) is used as the regularization method in which the continuum is enriched by the microstructure effects. Thus, the kinematics includes macrokinematics as well as microkinematics (Mindlin 1964, Germain 1973).

Equations (10) and (11) are two balance equations in the weak form which should be solved in the coupled second gradient model for every kinematically admissible virtual displacement field $u_{i}^{\star}$ and virtual pore water pressure field $p_{w}^{\star}$ :

$$
\begin{aligned}
& \int_{\Omega}\left(\sigma_{i j} \frac{\partial u_{i}^{\star}}{\partial x_{i}}+\sum_{i j k} \frac{\partial^{2} u_{i}^{\star}}{\partial x_{i} \partial x_{k}}\right) d \Omega= \\
& \int_{\Omega} G_{i} u_{i}^{\star} d \Omega+\int_{\Gamma_{\sigma}}\left(\bar{t}_{i} u_{i}^{\star}+\bar{T}_{i} D u_{i}^{\star}\right) d \Gamma, \\
& \int_{\Omega}\left(\dot{M} p_{w}^{\star}-m_{i} \frac{\partial p_{w}^{\star}}{\partial x_{i}}\right) d \Omega=\int_{\Omega} Q p_{w}^{\star} d \Omega+\int_{\Gamma_{\sigma}} \bar{q} p_{w}^{\star} d \Gamma,
\end{aligned}
$$

where $\Omega$ is the current solid configuration (volume), $G_{i}$ is the body force per unit volume, $\bar{t}_{i}$ is the external traction (classical) forces per unit area, $\bar{T}_{i}$ is an additional external (double) force per unit area that both $\bar{t}_{i}$ and $\bar{T}_{i}$ applied on a part $\Gamma_{\sigma}$ of the boundary of $\Omega, D u_{i}^{\star}$ is the normal derivative of $u_{i}^{\star}, \dot{M}$ is the time derivative of the water mass inside $\Omega, m_{i}$ is the mass flow, $Q$ is a sink term, and $\Gamma_{q}$ is the part of the boundary where the input water mass per unit area $q$ is prescribed. In addition, $\sum_{i j k}$ is the double stress, dual of the (micro) second gradient, which needs an additional constitutive law, and it has no link with the pore water pressure. It is defined with an elastic law (Mindlin 1964) as a function of (micro) second gradient of the virtual displacement where it depends on one elastic parameter $\mathrm{D}$ that the shear band width is proportional to this elastic parameter (Chambon et al. 1998, Kotronis et al. 2007).

\section{NUMERICAL SIMULATION OF EXCAVATION OF THE CONNECTING GALLERY}

In this section, the hydro-mechanical behavior of Boom clay during the excavation of Connecting gallery-one of the main galleries excavated in the 


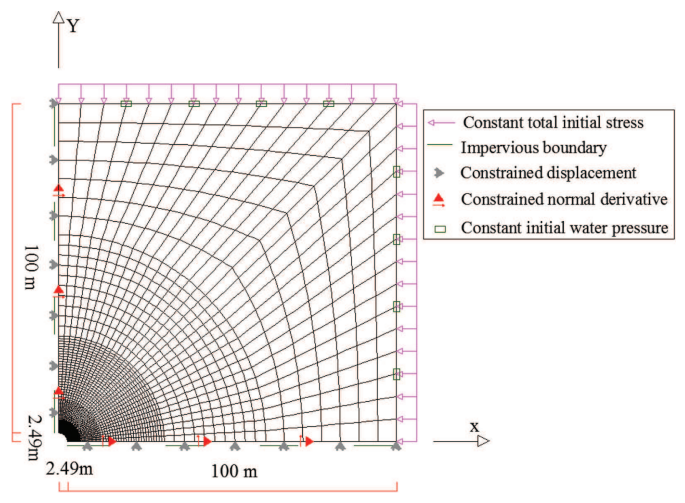

Figure 1. Geometry of the model.

underground research laboratory (URL) close to the city of Mol, Belgium, in order to study the feasibility of high-level nuclear waste disposal in the Boom Clay layer is analyzed. A quarter of the gallery is modeled by symmetry in the plain strain state using the Finite Element code, Lagamine, from the Université de Liège, and in the coupled hydro-mechanical conditions.

\subsection{The model's geometry and initial conditions}

The two-dimensional mesh geometry of the model is brought in figure 1 . The gallery has an over-excavated radius of $2.49 \mathrm{~m}$ taken into account $40 \mathrm{~cm}$ of the concrete lining and $9 \mathrm{~cm}$ of the convergence between the massive and the lining; the hydro-mechanical interface elements are considered on the interface between the massive and the lining. The initial boundary conditions are indicated in figure 1; the constrained normal derivative are given based on the fact of symmetry of the radial displacements around the symmetric boundaries (Zervos et al. 2001). The anisotropic initial stresses are applied as $\sigma_{y y}=4.5 \mathrm{MPa}$ and $\sigma_{x x}=$ $3.825 \mathrm{MPa}$. $\mathrm{MPa}$ which are decreased to $100 \mathrm{KPa}$ on the gallery wall during the excavation phase (1 day) and remain constant until the end of simulation (3.5 years). Furthermore, the initial pore water pressure is equal to $2.25 \mathrm{MPa}$ which there is a decrease up to the atmospheric pressure on the gallery wall during the excavation phase. Since the convergence of the massive due to the excavation is mostly taken place during the excavation phase, it is supposed that the contact between the massive and the lining is occurred by there and from then on, the water pressure remains constant (equal to the atmospheric pressure) only on the internal surface of the concrete lining.

\subsection{The model properties}

The model is analyzed using the frictional elastoplastic model with the Drucker-Prager yield surface and the second gradient method as it has been described in section 2. The applied flow model lets reproducing the existed transfers in porous media.
Table 1. Mechanical parameters of the model.

\begin{tabular}{llll}
\hline Parameter & Symbol & Value & Unit \\
\hline Young modulus & $E$ & 300 & $\mathrm{MPa}$ \\
Poisson ratio & $\vartheta$ & 0.125 & - \\
Specific mass & $\rho$ & 2682 & $\frac{\mathrm{kg}}{\mathrm{m}^{3}}$ \\
Initial friction angle & $\varphi_{0}$ & 8 & $\circ$ \\
Final friction angle & $\varphi_{f}$ & 18 & $\circ$ \\
Hardening/softening coefficient & $B_{\phi}$ & 0.001 & - \\
Initial cohesion & $c_{0}$ & 300 & $\mathrm{kPa}$ \\
Final cohesion & $c_{f}$ & 30 & $\mathrm{kPa}$ \\
Hardening/softening coefficient & $B_{c}$ & 0.01 & - \\
Dilatancy angle & $\Psi$ & 0 & $\circ$ \\
Second gradient elastic modulus & $D$ & 2000 & $\mathrm{~N}$
\end{tabular}

Table 2. Hydraulic parameters of the model.

\begin{tabular}{llll}
\hline Parameter & Symbol & Value & Unit \\
\hline Water permeability & $k_{w}$ & $3 \times 10^{-19}$ & $\mathrm{~m}^{2}$ \\
Specific mass of water & $\rho_{w}$ & $1 \times 10^{3}$ & $\frac{\mathrm{kg}}{\mathrm{m}^{3}}$ \\
Porosity & $\phi$ & 0.39 & - \\
Water compressibility & $\frac{1}{\chi_{w}}$ & $5 \times 10^{-10}$ & $\mathrm{~Pa}^{-1}$ \\
Van Genuchten parameter* & $m$ & 0.47 & - \\
Van Genuchten parameter* & $n$ & 1.887 & - \\
Van Genuchten parameter* & $P=\frac{1}{\alpha}$ & $7 \times 10^{6}$ & $\mathrm{kPa}$ \\
\hline
\end{tabular}

* Water retention curve and water permeability with reference to the Van Genuchten model (Van Genuchten 1980).

Tables 1 and 2 show the hydraulic and mechanical parameters of the material used in the modeling which have been chosen basically with reference to Bernier et al. (2007). The cohesion softening and friction angle hardening are then applied. In addition, several simulations were performed in the framework of parametric study to better define the essential parameters for modeling the strain localization phenomenon like cohesion softening (parameters $c_{f}$ and $B_{c}$ ) and second gradient elastic modulus $D$. The second gradient parameter has a significant influence on the width of the shear strain localization bands. In addition, the study showed the role of the cohesion softening in order to initiate the strain localization around the gallery and consequently, the initial cohesion was supposed to be divided by factor 10 to give the final cohesion as it is brought in table 1 .

\subsection{Analysis of the results}

The evolution of the shearing bands during the excavation and afterwards are brought in figure 2 in terms of total deviatoric strain for four different time during the simulation ( 0.6 day, 1 day as the end of excavation phase, 100 days, and 3.5 years).

As it is observed in figure 2 , the evolution of the bands and extension of the EDZ is quite limited to 

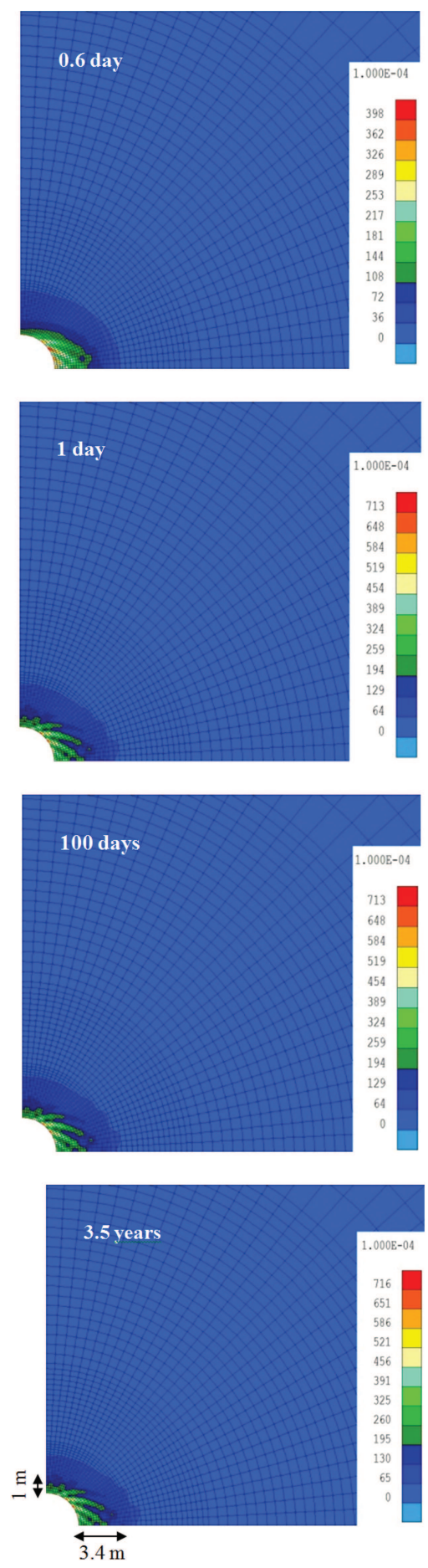

Figure 2. Total deviatoric strain.

the excavation phase since after one day, the activity of the bands of localization is not evident. This phenomenon is better clarified in figure 3 where the evolution of the bands of localization are brought in
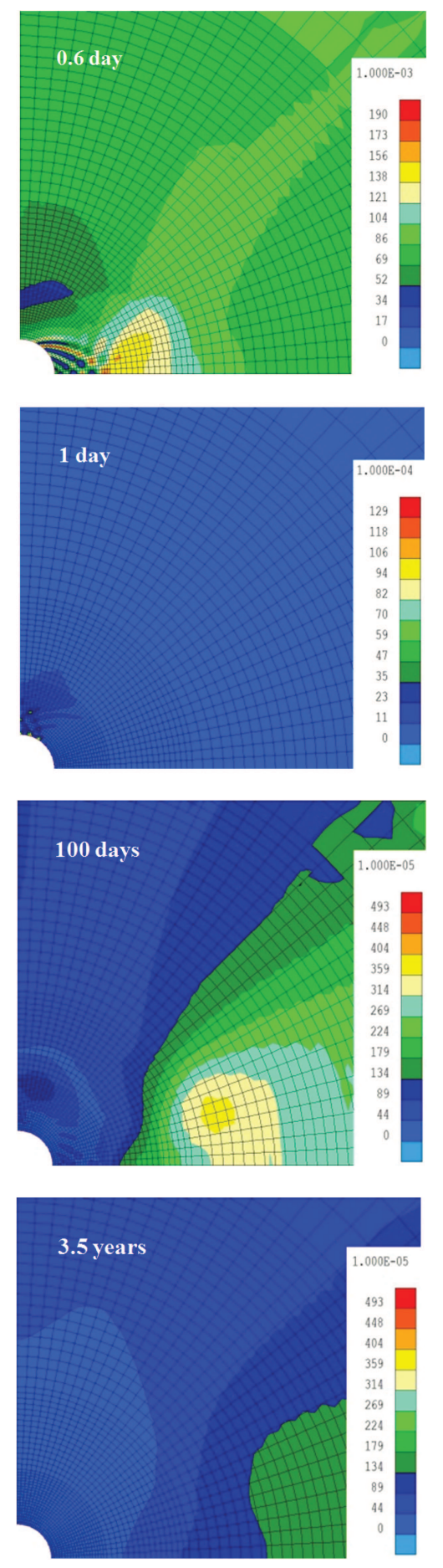

Figure 3. Increment of deviatoric strain.

terms of the increment of deviatoric strain that it is not showing any obvious activity of the bands after the end of excavation phase. Therefore, the extension of the EDZ can be estimated as about $3.4 \mathrm{~m}$ horizontally 


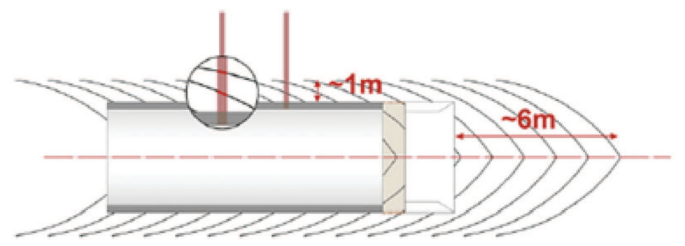

Figure 4. Vertical cross-section of observed fractures around the Connecting gallery (Mertens et al. 2004).

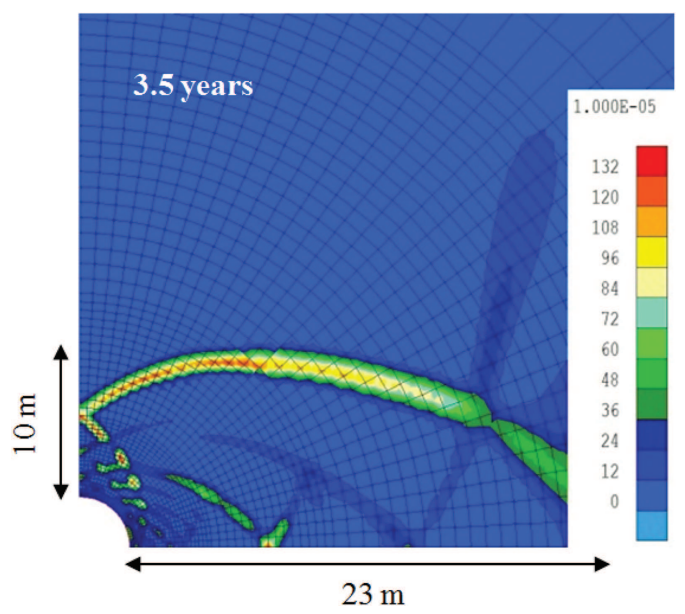

Figure 5. Increment of deviatoric strain; Gallery without the lining.

and $1 \mathrm{~m}$ vertically (figure 2), which is similar to the in-situ observations regarding the radial extension of the EDZ that is about one meter (figure 4).

Moreover, the study aimed to highlight the essential influence of the concrete lining in decreasing the extension of the damaged zone and the convergence of the massive around the gallery. Figure 5 shows the increment of deviatoric strain for the case without the lining after 3.5 years. The bands of localization are still active at the end of simulation which can confirm a larger extension of the EDZ comparing to the case with the lining (figure 2). In fact, adding the lining can prevent the high amount of convergence in the vicinity of the gallery and thus, the evolution of the shearing bands would be more limited. On the other words, the contact between the massive and the lining is launched to be taken place during the excavation establishing the contact pressure on the the interface elements. This phenomenon has a significant role in decreasing the extension of EDZ. Figure 6 shows the normal contact pressure on the interface elements at the end of excavation phase.

Figure 7 shows the evolution of pore water pressure for three cross-sections - horizontal, inclined $\left(45^{\circ}\right)$ and vertical - in function of the distance from the gallery wall, and for different times during the simulation. As it is observed, there is an increase pattern in the evolution process of water pressure in the horizontal cross-section, which is interacted by the decrease

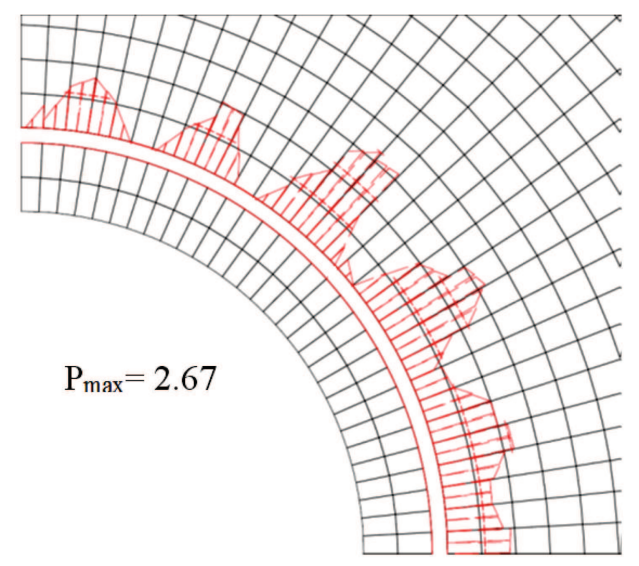

Figure 6. Diagram of the normal contact pressure on the interface contact elements.

of water pressure (suction tendency in the vicinity of the gallery after the end of excavation) in the vertical section. The reason can be found in the deformation pattern of the massive which makes the contact to be taken place in the horizontal axis sooner than the vertical one. Consequently, the internal water flow is conducted towards the horizontal axis from the vertical one which can justify the pattern of evolution of pore water pressure. In addition, the appearance of localization bands and its development is reflected in the evolution of pore water pressure with some ups and downs, specially in the horizontal axis where the shear bands are more developed.

\section{CONCLUSIONS}

In the framework of deep disposal of high-level nuclear waste in the potential geological formations, Boom clay is studied as a potential host rock due to its favorable properties, among which its very low hydraulic conductivity. Since the creation of an Excavated Damaged Zone (EDZ) around the galleries is inevitable, the initial properties of the host-rock may be locally modified during the excavation which can be important in the framework of long-term performance of these underground repositories. In this way, studying the evolution of fracture network in the framework of strain localization subsequent to rock's damage permits a better understanding of the rock's hydraulic conductivity changes.

The excavation process of Connecting gallery in the Boom clay host-rock in the underground research laboratory in Mol; Belgium; was modeled hydromechanically. The results have provided realistic information on the extension of the Excavation Damaged Zone and strain localization bands around the gallery during and after the excavation. Moreover, the important influence of the concrete lining around the galley in the Boom clay host-rock was emphasized in order to decrease the extension of the damaged zone 

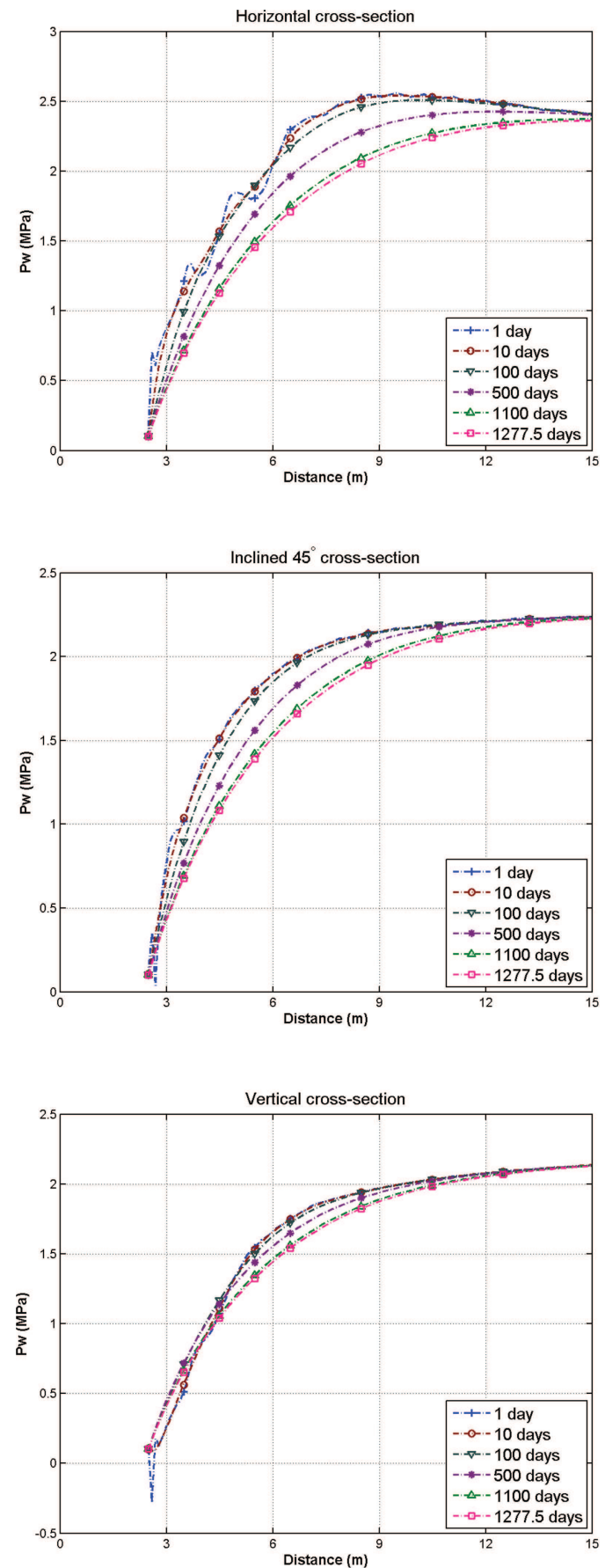

Figure 7. Pore water pressure evolution.

and convergence of the massive. A discussion on the contact pressure on the interface elements between the massive and the lining has also confirmed the role of the lining. The convergence pattern of the massive towards the lining could also be observed in the process of evolution of pore water pressure in the vicinity of the gallery.

\section{ACKNOWLEDGEMENT}

The authors would like to thank the Belgian National Agency for Radioactive Waste and enriched Fissile Material; ONDRAF/NIRAS; for their financial support in this study.

\section{REFERENCES}

Barnichon, J.-D. 1998. Finite element modelling in structural and petroleum geology. $\mathrm{Ph}$. D. thesis, Université de Liège.

Bernier, F., X. L. Li, \& W. Bastiaens 2007. Twenty-five years' geotechnical observation and testing in the tertiary boom clay formation. Géotechnique 57(2): 229-237.

Chambon, R., D. Caillerie, \& N. El Hassan 1998. Onedimensional localisation studied with a second grade model. European Journal of Mechanics-A/Solids 17(4): 637-656.

Chambon, R., D. Caillerie, \& T. Matsuchima 2001. Plastic continuum with microstructure, local second gradient theories for geomaterials: localization studies. International Journal of Solids and Structures 38(46): 85038527.

Collin, F., S. Levasseur, \& R. Chambon 2009. Numerical post failure methods in multiphysical problems. European Journal of Environmental and Civil Engineering 13(7-8): 983-1004.

Germain, P. 1973. The method of virtual power in continuum mechanics. part 2: Microstructure. SIAM Journal on Applied Mathematics 25(3): 556-575.

Kotronis, P., F. Collin, P. Bésuelle, R. Chambon, \& J. Mazars 2007. Local second gradient models and damage mechanics: 1d post-localization studies in concrete specimens. In Bifurcations, Instabilities, Degradation in Geomechanics, pp. 127-142. Springer.

Lenoir, N., M. Bornert, J. Desrues, P. Bésuelle, \& G. Viggiani 2007. Volumetric digital image correlation applied to x-ray microtomography images from triaxial compression tests on argillaceous rock. Strain 43(3): 193-205.

Mertens, J., W. Bastiaens, \& B. Dehandschutter 2004. Characterisation of induced discontinuities in the boom clay around the underground excavations (urf, mol, belgium). Applied clay science 26(1): 413-428.

Mindlin, R. 1964. Micro-structure in linear elasticity. Archive for Rational Mechanics and Analysis 16(1): 51-78.

Rice, J. R. 1976. The localization of plastic deformation. Division of Engineering, Brown University.

Van Genuchten, M. T. 1980. A closed-form equation for predicting the hydraulic conductivity of unsaturated soils. Soil Science Society of America Journal 44(5): 892-898.

Zervos, A., P. Papanastasiou, \& I. Vardoulakis 2001. Modelling of localisation and scale effect in thick-walled cylinders with gradient elastoplasticity. International Journal of Solids and Structures 38(30): 5081-5095. 